\title{
Analysis of the Interference Signal of the Distributed Optical Fiber Sensing Based on DSP*
}

\author{
Tingting Du ${ }^{1}$, Xiong Wan ${ }^{1,2 \#}$, Zhimin Zhang ${ }^{1}$, Huming Zhang ${ }^{1}$, Qing Zhang ${ }^{1}$ \\ ${ }^{1}$ Key Laboratory of Nondestructive Testing(Ministry of Education), Nanchang Hangkong University, Nanchang, Jiangxi, China \\ ${ }^{2}$ Key Laboratory of Spatial Active Opto-electronic Techniques, Shanghai Institute of Technical Physics, \\ Chinese Academy of Sciences, Shanghai, China \\ Email: \#nchundt114@126.com
}

Received 2013

\begin{abstract}
In this paper, the author analyzes characteristics and extracting method of interference signal of the distributed optical fiber sensing. In the distributed optical fiber sensing, realizing alarm and positioning function only through the crosscorrelation operation will increase the load of the system, can make misinformation rate of the system be improved greatly. Therefore, before the localization algorithm, adding a interference signal feature recognition is very necessary, can reduce unnecessary operation loss and reduce the load of the system, also reducing the number of the false positives.
\end{abstract}

Keywords: The Optical Fiber Sensing; Mach-zehnder Interferometer; Energy; Interference Signal; Signal Recognition

\section{Introduction}

With the development of optical fiber communication technology, the optical fiber sensing technology draws a great attention, and is widely used in the earthquake monitoring[1,6,7], temperature alarm [2] and becomes one of the most important technology in information society, and has huge market potential. Optical fiber sensor uses fiber itself sensitivity characteristics work. When the light from the laser light source spreads in the fiber, if external factors such as stress, temperature, electric field and magnetic field change, the characteristic parameters such as the light wave amplitude, phase, wavelength and polarization state will change[3].Therefore, the optical fiber sensing technology draws a great attention, be researched and applied in almost every field .

This paper uses the sensor that is based on machzehnder interferometer [5,8]. The biggest advantage of the interferometric sensor is that interference detecting sensitivity is extremely high. In mach-zehnder interferometer fiber-optic sensor, alarm and positioning are two main functions of the distributed optical fiber sensing system. The pretreatment of the interference signal is of great significance to improve the alarm accuracy and the

*This work was supported by Chinese Natural Science Foundation (grant 61271397 ), Jiangxi provincial Natural Science Foundation (grant 20122 BAB202009), Jiangxi provincial education department Science and Technology Foundation (grant GJJ12408) and preferentially funded by the "Hundred Talents Plan" of the Chinese Academy of Sciences.

${ }^{\#}$ Corresponding author. positioning accuracy. Sorting the interference signal that is gathered by Digital Signal Processor (DSP), establishing corresponding mode, filtering useless information and noise, selecting and extracting needed information, can greatly improve the sensor system alarm and positioning accuracy. Based on the short time analysis method, the paper discusses and analyzes short-term energy of identification method.

\section{Experimental System}

\subsection{Experimental System Structure}

The experiment system schematic diagram as shown in Figure 1, uses DFB laser, its wave length is $1550 \mathrm{~nm}$, the line width is 2MHZ. The light that the DFB laser produces is divided into two beam of light by the coupler 1. One of the two beam of light through the fiber coupler 3 goes into MZI, spreading along the clockwise in the interferometer, occurring interference in the coupler 4. Finally, half of light power of the coupler 2 is received by photoelectric detector PIN1.The other of the two beam of light spreads along the counterclockwise in the interferometer, occurring interference in the coupler 3. Finally, half of light power of the coupler 3 is received by photoelectric detector PIN2.

When vibration or pressure signal applies to optical fiber interference arm, geometric size and refractive index parameters of fiber will change, resulting in light wave phase change. Through photoelectric detector, the 


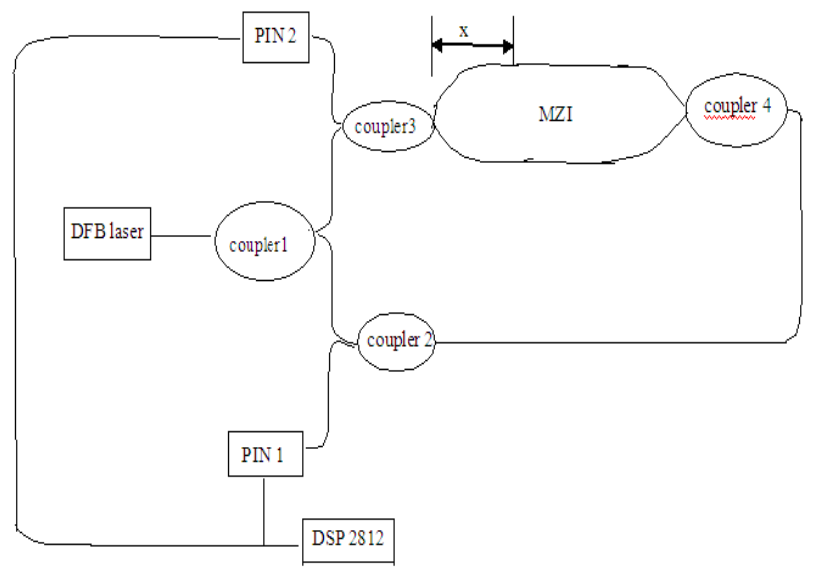

Figure 1. Experimental of the interference signal analysis.

change of intensity of interference light will turn into a change of electrical signal. Through detecting and recognizing these electrical signals, we can realize alarm of the invasion signal. The experiment uses Digital Signal Processor (DSP) process signal. Because DSP has strong graphic display ability, not only can show the original signal graphics, also can display FFT spectrum diagram of signal. Because DSP2812 in a period can complete 32-bit * 32-bit multiplicative additive operation. It makes the processing speed of algorithm in DSP's chip faster. Therefore, we use DSP do operation for interference signal.

\subsection{System Positioning Principle}

In Figure 1, supposing the optical fiber length is L meters between coupler 3 and coupler 4 , If in a moment this position that has $\mathrm{x}$ meters from coupler 3 happens disturbance. The disturbance signal will respectively spread along the clockwise and anti-clockwise direction. The time that the light spreading along Clockwise direction and arriving to coupler 2 takes is $t_{1}$. The time that the light spreading along counterclockwise direction and arriving to coupler 3 takes is $t_{2}$. By measuring the time difference, we can get vibration point position so as to realize positioning[4].

$$
\begin{aligned}
& t_{1}=\frac{(L-x) n}{c} \\
& t_{2}=\frac{x \times n}{c}
\end{aligned}
$$

\section{Experimental Installment}

\subsection{Experiment}

Outputed signal of the optical fiber sensor in addition to containing vibration interference signal, also contains noise interference signal. In order to avoid noise signal, to improve the effectiveness of the subsequent testing, it is necessary to identify disturbance signal and noise signal. This experiment mainly analyses the characteristics of two kinds of actual signal that are disturbance signal and noise signal.

In the experiment, we use the short-term energy analysis method. Short-term energy analysis is based on frame and window for signal. As such, the basic concept for processing interference signal is that divide discrete interference signal into a certain length unit to process, also is "short" processing method.

If the discrete sequence that analog signal outputed by the sensor through $\mathrm{AD}$ change is $x(i), i=0,1,2 \ldots m$, the short-term energy $S(n)$ calculation formula is

$$
S(n)=\sum_{i=-\infty}^{+\infty}(x(i) w(n-i))^{2}=\sum_{i=n-N+1}^{n} x^{2}(i) w^{2}(n-i)
$$

The $w(n)$ represents sliding window function, $\mathrm{n}=0$, $1, \ldots . . .(N-1)$..

Particularly, when the window function is rectangular window function, the short-term energy $S(n)$ calculation formula is

$$
S(n)=\sum_{i=n-N+1}^{n} x^{2}(i)
$$

The original signal diagram is shown in Figures 2-3. Vibration interference signal diagram and noise signal diagram have obvious difference. The short-term energy of signal chart is shown in Figures 4-7. Vibration interference signal of the short-term energy diagram and noise signal of the short-term energy diagram also have obvious difference.

\subsection{Experimental Analysis}

The experiment system of the AD sampling rate is 100 $\mathrm{KHZ}$, every frame sampling point is 800 . Every frame signal of the short time average energy is shown in Table 1.

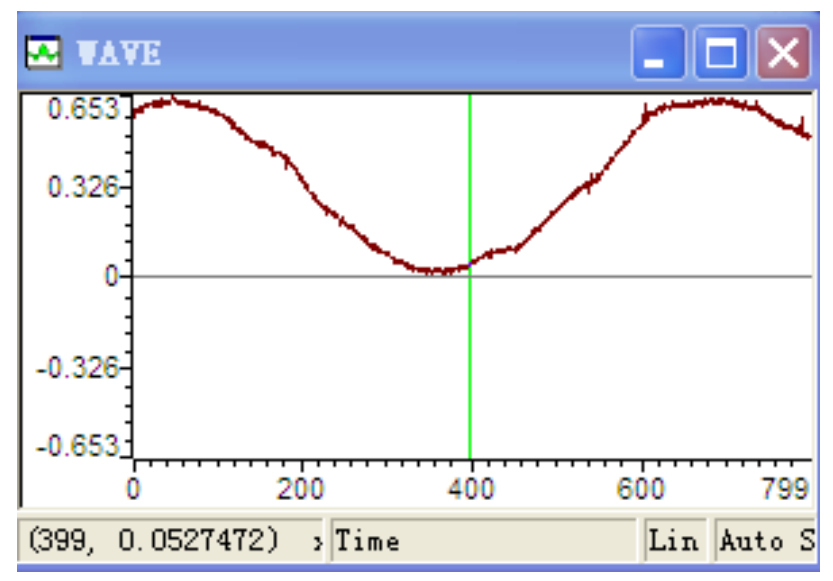

Figure 2. Vibration interference signal original diagram. 


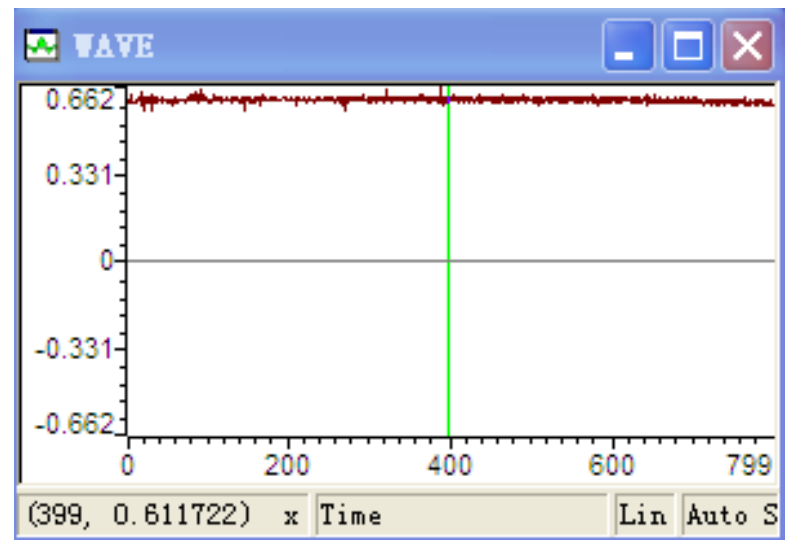

Figure 3. Noise interference signal original diagram.

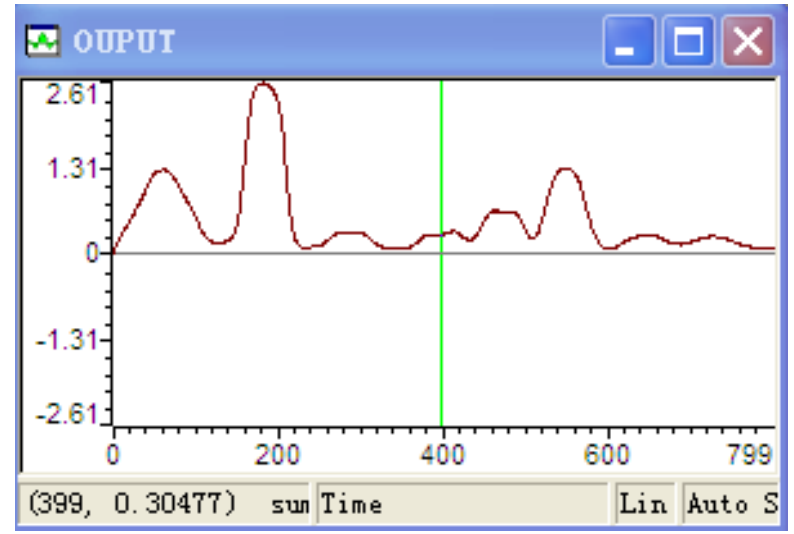

Figure 4. Vibration interference signal of the short-term energy diagram.

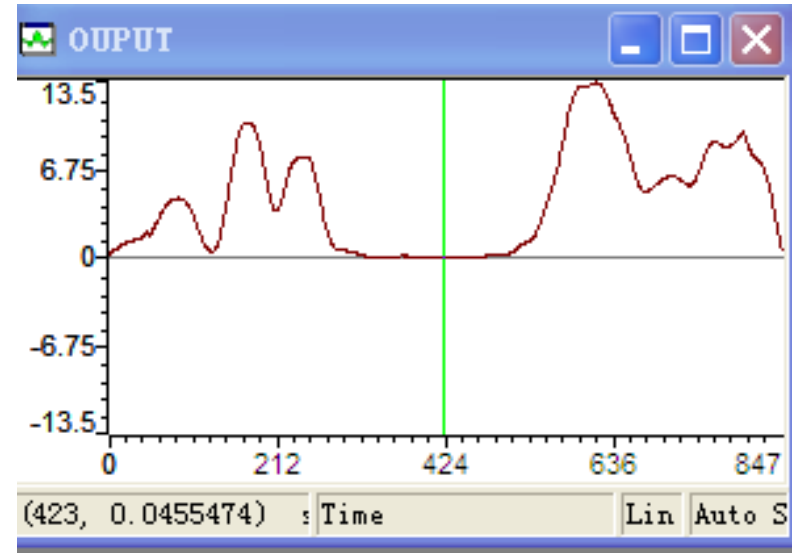

Figure 5. Vibration interference signal of the short-term energy diagram.

From the above, we can be seen that we can use the short time average energy distinguish between the vibration signal and noise signal. When the short time average energy is smaller than a certain threshold (for example 12), we judge it as vibration signal. When the short time average energy is greater than a certain threshold, we judge it as noise signal.

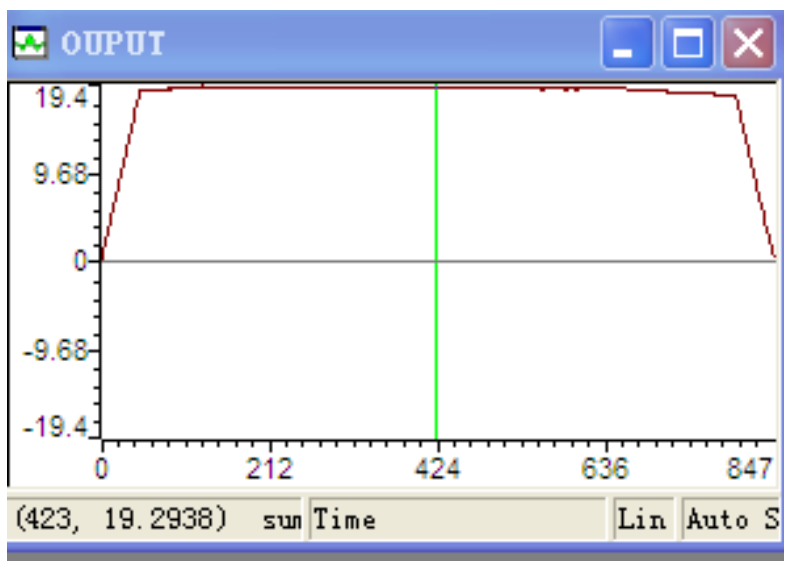

Figure 6. Noise interference signal of the short-term energy diagram.

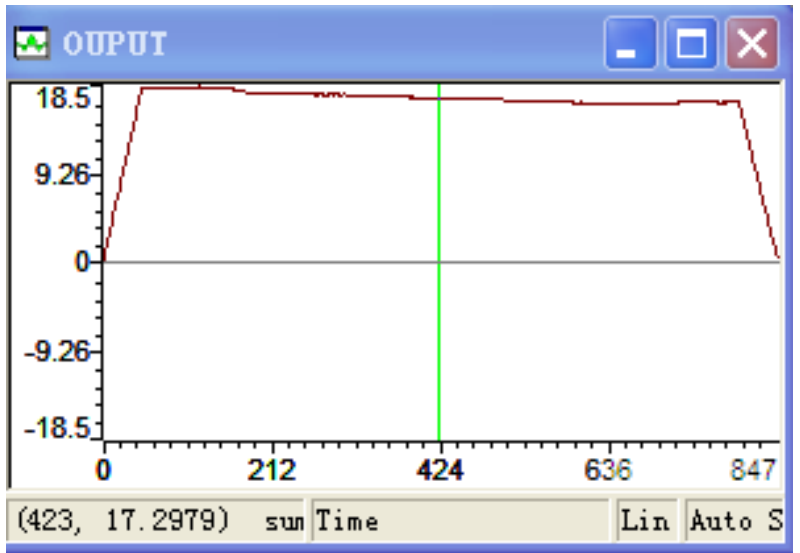

Figure 7. Noise interference signal of the short-term energy diagram.

Table 1. Short time average energy.

\begin{tabular}{lll}
\hline \multicolumn{1}{c}{ Signal types } & \multicolumn{1}{c}{ Frame number } & $\begin{array}{c}\text { Short time average } \\
\text { energy }\end{array}$ \\
\hline vibration signal & $800100 \mathrm{XH} 0$ & 0.5027 \\
vibration signal & $800100 \mathrm{XH} 1$ & 3.0623 \\
vibration signal & $800100 \mathrm{XH} 2$ & 0.4807 \\
vibration signal & $800100 \mathrm{XH} 3$ & 9.2760 \\
vibration signal & $800100 \mathrm{XH} 4$ & 6.6546 \\
Noise signal & $800100 \mathrm{X} 0$ & 17.9425 \\
Noise signal & $800100 \mathrm{X} 1$ & 16.8052 \\
Noise signal & $800100 \mathrm{X} 2$ & 16.4081 \\
\hline
\end{tabular}

\section{Conclusions}

This experiment completes the signal of characteristic identification by using DSP. The signal feature recognition is an important part of signal pretreatment of the distributed optical fiber sensing. This experiment analyzes signal characteristics in time domain. The technology filters undesired signal, greatly reducing the unnec- 
essary operation loss, decreasing the system load, and at the same time, improving the alarm positioning accuracy.

\section{REFERENCES}

[1] D. Lgardner and S. Lgarrett, "Fiber Optic Seismic Sensor,” Fiber Optic and Laser sensor V, Vol. 1987, No. 838, pp. 271-278

[2] C. X. Ao, K. X. Jin, T. Li, et al., "Mach-Zehnder Optical Fiber Temperature sensor research," Journals of Shanxi Technology University, Vol. 22, No. 6, 2004, pp.104-107.

[3] Y. He and D. M. Liu, "Optical Fiber Sensor Phase Demodulation Technology Based on Labview,” 2006, Vol. 19, No.1, pp. 94-97.

[4] J. Tang, "Fiber Optic Interferometer Phase Detection Research Based on Mach-ZeHnder,” Sensing Technology,
Vol. 6, No. 2, 2000, pp. 96-100.

[5] E. UDD, "Fiber Optical Sensors: An Introduction for Engineers and Scientists," John Wiley \& Sons, Canad, 1991.

[6] J. P. Dakin, "Distributed Anti-Stokes Ratio Thermometry” 3rd International Conference on Optical Fibre Sensors, San Diego,1985.

[7] T. Ohmachi and H. Matsumot, "Sea Water Pressure Included by Seismic Ground Motions and Tsunami,” Proceeding of International Tsunami Symposium, 2001, pp. 595-609.

[8] A. A. Chtcherbakow, P. L.Swart and S. J. Spammer “A Fiber Optic Disturbance Location Sensor Using Modified Sagnac and Mach-Zehnder Interferometers,” Proceeding of 12th Optical Fiber Sensor, 1997, Vol. 16, pp. 516-519. 